\title{
Thyroid and Cardiovascular Disease: A Focused Review on the Impact of Hyperthyroidism in Heart Failure
}

\author{
Rafay Khan ${ }^{\text {a, d }}$, Saad Sikanderkhel ${ }^{\mathrm{a}}$, Junhong Gui ${ }^{\mathrm{a}}$, Abdul-Razaq Adeniyi ${ }^{\mathrm{a}}$, Kimberly O’Della, \\ Marc Erickson ${ }^{\mathrm{a}}$, Juan Malpartida ${ }^{\mathrm{a}}$, Zarmina Mufti ${ }^{\mathrm{b}}$, Taiba Khan ${ }^{\mathrm{c}}$, Harris Mufti ${ }^{\mathrm{b}}$, \\ Saif Aldeen Al-Adwan ${ }^{a}$, Diana Alvarez ${ }^{\text {, }}$, Joshua Davis ${ }^{\text {, }}$ \\ Joseph Pendley ${ }^{\mathrm{a}}$, Dharmendra Patel ${ }^{\mathrm{a}}$
}

\begin{abstract}
The association between thyroid hormones and cardiovascular conditions has been well studied, specifically, the effects of hypothyroidism on cardiomyopathy, and hyperthyroidism with arrhythmias. Nonetheless, an explicit correlation between hyperthyroidism and cardiomyopathy has yet to be established. Medical databases MEDLINE and PubMed were accessed and queried as primary sources for data acquisition. Search criteria consisted of "hyperthyroidism", "heart failure", and "thyroid and cardiovascular system", which allowed the retrieval of relevant and recent works. From these sources, a consensus was developed and employed to yield an updated review of the etiology of heart failure in the setting of hyperthyroidism. It is rare for patients with hyperthyroidism to remain in a chronic hyperthyroid state, making it difficult to analyze subsequent long-term effects on the cardiovascular system. Related to heart failure, some studies have demonstrated no change in ejection fraction, while others have shown an acute change along with diastolic dysfunction, with or without an underlying rhythm abnormality. Further investigation is warranted to elucidate the mechanism driving such cardiac dysfunction, and whether it is due to vascular changes, tachyarrhythmias, or myocyte remodeling and fibrosis. The intent of this review article is to improve our understanding of how a hyperthyroid state affects cardiovascular function. An enhanced understanding of the effects on cardiovascular physiology will afford physicians the ability to provide more comprehensive care in consideration of both endocrine and cardiovascular pathologies.
\end{abstract}

Keywords: Heart failure; Thyroid; Hyperthyroidism; Cardiovascular

Manuscript submitted February 10, 2020, accepted February 21, 2020

aDepartment of Cardiology, University of Tennessee at Chattanooga, $975 \mathrm{E}$ 3rd Street, Chattanooga, TN 37403, USA

'Frontier Medical College, Karakoram Highway, Mansehra Rd, Abbottabad, Khyber Pakhtunkhwa, Pakistan

'Bucknell University, 701 Moore Ave, Lewisburg, PA 17837, USA

${ }^{\mathrm{d} C o r r e s p o n d i n g ~ A u t h o r: ~ R a f a y ~ K h a n, ~ D e p a r t m e n t ~ o f ~ C a r d i o l o g y, ~ U n i v e r s i t y ~}$ of Tennessee at Chattanooga, 975 E 3rd Street, Chattanooga, TN 37403, USA.

Email: rafay.t.khan@gmail.com

doi: https://doi.org/10.14740/cr1034

\section{Introduction}

The thyroid gland secretes thyroid hormones (THs), triiodothyronine (T3) and thyroxine (T4), which are formed from iodine, and influence metabolic rate and protein synthesis. Regulation of these hormones is driven by the anterior pituitary gland via the secretion of thyroid stimulating hormone (TSH), which itself is regulated by the hypothalamus via thyrotropin releasing hormone (TRH). THs incite multiple effects on the heart and peripheral vasculature. Notably, the intracellular cardiac effects of THs occur via two mechanisms: genomic or nongenomic, with most effects exerted via the genomic pathway.

In the genomic pathway, $\mathrm{T} 3$ binds to thyroid responsive elements in the promoter segment of target genes [1]. This activates gene expression, specifically via messenger RNA that encodes for proteins with variable tissue-specific responses. Via this mechanism, synthesis of cardiac protein is stimulated, leading to myocardial hypertrophy and dysfunction [1]. In contrast, the non-genomic pathway affects changes in the cardiac myocyte plasma membrane and cytoplasmic organelles, which may result in alterations within the intracellular signaling pathways in cardiac and smooth muscle cells [1]. The effects of these two pathways working in tandem have been postulated as the mechanism driving cardiac dysfunction in a hyperthyroid state; this has been shown to be present in 5-10\% of the population, with a higher prevalence in the elderly [2-4].

Heart failure has been diagnosed in about 5.8 million patients in the USA [2, 5]. Several studies have analyzed the association between heart failure and hypothyroidism, but few studies have assessed the correlation between hyperthyroidism and cardiac pathologies, with exception of atrial fibrillation and tachyarrhythmias. This review presents an analysis of various studies addressing the correlation between these medical conditions.

\section{TH Physiological Effects on Vasculature}

T3 and T4 have been shown to exert physiological effects on multiple organ systems, including the vascular system. T3 directly affects vascular smooth muscle cells and promotes relaxation $[6,7]$. It has been postulated that $\mathrm{T} 3$ reduces expression of angiotensin II type 1 receptors, and thereby reduces the contractile response to angiotensin II $[6,8]$. T3 also activates 
the phosphoinositol 3-kinase (PI3-K)/Ak-mediated endothelial nitric oxide synthase signaling pathway, which stimulates the production of nitric oxide (NO) $[6,9,10]$. NO is vital for vascular homeostasis and affects dilator tone. The consequence of these effects is reduced vascular constriction [6].

Additional reports have shown that T3 promotes angiogenesis, and may increase the density of coronary arterioles, thus impacting the process of myocardial ischemic reconditioning $[6,7,11,12]$. Patients with hypothyroidism may develop increased diastolic blood pressure, via decreased endotheliummediated relaxation and vascular compliance $[6,13]$. Conversely in hyperthyroidism, there is a decrease in peripheral vascular resistance, increased blood volume, and increased venous return $[6,14]$. These changes may lead to development of heart failure secondary to a high level of output.

\section{TH Effects on Cardiovascular Physiology}

THs affect myocardial contractility, total peripheral resistance and heart rate. They enhance myocardial contractility by upregulating calcium handling and myosin heavy chain isoforms, and stimulating the beta adrenergic system [7, 15, 16]. Hyperthyroidism has been shown to incite arrhythmias, vascular changes, and myocardial remodeling. These changes can decrease cardiac output and promote the development of heart failure. Notwithstanding, current literature analyzing the relationships between myocyte function and global cardiac function in the setting of hyperthyroidism is limited [17]. It has been demonstrated that excess TH may result in tachycardia, widened pulse pressure, increased cardiac output, and decreased total peripheral resistance. Although these effects have been well analyzed, there is no established consensus regarding mechanism of action $[7,17]$.

The mechanism of action of THs is prominently a result of their action on nuclear thyroid hormone receptors (TR). TR-alpha, the main TR isoform receptor of the cardiovascular system, is activated by T3 [6, 7, 18-20]. TR cardiomyocyte growth is due to phosphorylation of PI3-K, protein kinase B, and mammalian target of rapamycin (mTOR), which enhance developmental processes, such as sarcomere protein translation $[6,21-23]$. The activation of these signaling pathways leads to changes in gene expression, which is comparable to physiologic mechanism of cardiac hypertrophy [6].

There is evidence that serum levels of T4 and T3 are frequently decreased in heart failure patients, and decreased thyroid function may contribute to systolic and diastolic dysfunction [6]. The data relating to a prolonged hyperthyroid state and heart failure are less consistent. Numerous other conditions have also been correlated with thyroid function, including pericarditis, cardiac tamponade, tachycardia, atrioventricular block, pericardial effusion, sinus bradycardia, torsade de pointes, cardiomyopathy, endothelial dysfunction, hypertension, and dyslipidemia [6].

\section{A Brief Look at Hypothyroidism and Cardiology}

Hypothyroidism, a condition with low levels of T4 and T3 and compensatory high levels of TSH, can result in diastolic hypertension, sinus bradycardia, and heart failure. A brief overview of the causal relationship between hypothyroidism and cardiovascular disease will be reviewed prior to addressing the lesser-studied association seen with hyperthyroidism. Chronic hypothyroidism has been shown to increase the risk of atherosclerosis owing to its association with hyperlipidemia [6]. Secondly, electrocardiogram (ECG) changes typically found in hypothyroid states include sinus bradycardia, prolonged QTc and atrioventricular block. A combination of these findings has been demonstrated an increased risk of coronary artery disease (CAD). Other factors associated with hypothyroidism include endothelial dysfunction, decreased nitric oxide, and subsequently decreased vascular relaxation. These factors have similarly been seen in cases of subclinical hypothyroidism, where TSH levels are high with normal T4 and T3 levels. This condition has been correlated with left and right ventricular systolic and diastolic dysfunction and will be reviewed later.

For hypothyroid patients with CAD, lower doses of levothyroxine should initially be utilized, with a gradual increase in dose. Physicians may consider starting treatment with levothyroxine $12.5 \mu \mathrm{g}$ orally daily and increasing the dose after 6 weeks, to lower the peripheral vascular resistance and thus ameliorate the myocardial ischemia to achieve a euthyroid state [6]. Many articles have analyzed the association with low or subclinical thyroid states and cardiac conditions, but few have looked into the effects of hyperthyroidism, along with prognosis and therapy. This article will further focus on hyperthyroidism and the associated cardiovascular conditions.

\section{An Overview of Hyperthyroidism and Cardio- vascular Entities}

Typically a hyperthyroid state is characterized as a low TSH level associated with elevated free T4 (FT4). Some of these changes can be seen in iatrogenic thyroid disease, thyroiditis, nodular thyroid disease, Grave's disease, and thyroid storm. The workup includes TSH, T3 and FT4 in asymptomatic or symptomatic patients with suspected hyperthyroidism. If TSH is low, it is recommended to recheck the TSH level, obtain thyroid peroxidase antibodies, and thyroid stimulating immunoglobulin (TSI) levels.

Studies in patients with hyperthyroidism have demonstrated that an increase in TH results in reduced peripheral vascular resistance, elevated heart rate, and subsequently increased cardiac output [1]. The decrease in vascular resistance results in low renal perfusion, which arouses the renin-angiotensinaldosterone system (RAAS), and affects an increase in total blood volume. These changes may subsequently lead to a state of increased preload, decreased afterload, and increased stroke volume $[1,24]$. T3 has been shown to further stimulate the synthesis of renin in the liver itself, yielding increased cardiac levels of renin and angiotensin II. In the setting of hyperthyroidism, there is also a direct increase in the expression of angiotensin II receptors within the myocardium [1, 25]. Additionally, these changes can increase the release of atrial natriuretic peptide, which further enhances the level of vaso- 
dilation $[1,24]$

Upon review of the literature, the most common cardiac phenomenon noted in a state of hyperthyroidism is tachycardia, which often manifests in an irregularly irregular rhythm. Prolonged or exceedingly rapid tachyarrhythmia may lead to heart failure; and this form of tachycardia-induced cardiomyopathy results in decreased diastolic filling time and increased filling pressures.

Regarding atrial fibrillation, therapy is based on established guidelines, while also affecting the return to a euthyroid state. If in euthyroid state, the patient is more likely to remain in normal sinus rhythm. Notably, sinus tachycardia remains the most common arrhythmia in hyperthyroidism [6]. Heering et al [26] found that in a population-based study examining elderly patients, those in the lowest quartile of normal range of serum TSH had double the risk for atrial fibrillation compared with those who were in the highest quartile. Out of all cases of atrial fibrillation, only $1 \%$ of the patients have been shown to be secondary to hyperthyroidism; however, atrial fibrillation is the only cardiac condition that has been consistently associated with hyperthyroidism [6].

\section{Hyperthyroidism and Heart Failure}

Heart failure represents the initial clinical presentation in about $6 \%$ of patients with hyperthyroidism, with half having left ventricular (LV) dysfunction [27]. There are a limited number of studies that examine cardiac function and decompensation in the setting of hyperthyroidism; many of these study results provide conflicting data.

Hyperthyroidism has been shown to result in hemodynamic changes with a reduced ejection fraction (EF) and cardiac output due to a decrease in myocardial contractile reserve. One study was able to demonstrate the long-term effect of hyperthyroidism on LV remodeling, cardiac function, myocyte function, and tissue remodeling in hamster models [17]. In this study, one group was subject to receive thyroid-containing pellets and was compared to an untreated control group. After 1 month, the thyroid-treated group demonstrated a significant elevation in resting heart rate. After 4 months, there was significant depression in their EFs. Furthermore, between 6 and 10 months of treatment, there was severe reduction in the measured EF [17]. Notably, there was no difference in left ventricle internal dimension (LVId) during diastole after 1 month of treatment, but by 2 months there was a significant increase in left ventricular internal dimension in diastole (LVIDd) [17]. Despite preservation of left ventricle posterior wall diameter (LVPWd), progressive chamber enlargement led to diastolic wall stress with an increased LVIDd/LVPWd ratio by 6 months in the TH treatment group [17]. Compared with untreated hamsters, those treated were found to have a $60 \%$ increase in left ventricular fibrosis, primarily located within the perivascular space and cardiac interstitium; myocyte necrosis with replacement fibrosis was not found [17]. Overall, these findings in hamster models demonstrate deleterious cardiac remodeling, including myocyte lengthening, chamber dilation, decreased relative posterior wall thickness, increased wall stress, and increased LV fibrotic deposition with sustained hyperthyroid- ism, leading to systolic and diastolic dysfunction evidenced by echocardiogram and hemodynamics [17]. These findings have not been thoroughly researched in human patients; however, as human patients typically present with more profound symptoms, which lead to earlier diagnosis and treatment. Early treatment of most patients with hyperthyroidism may prevent these deleterious effects that were seen in hamster models. Most patients convert to a euthyroid state prior to any cardiac dysfunction or cardiac remodeling occurs.

Another study analyzing 2,225 patients assessed TSH levels measured at baseline and at 6-month intervals throughout a 5 -year study. TSH levels were correlated with the thyroid state of the patient, diagnosis of diastolic heart failure, and prognostic effect, with the overall risk of mortality being evaluated. The study revealed that abnormal thyroid function in patients with symptomatic heart failure and $\mathrm{EF}<35 \%$ was associated with an increased risk of death [2]. Furthermore, mean baseline EF was similar across the various thyroid groups. Repeat $\mathrm{EF}$ assessments at 12 and 30 months demonstrated an increase in mean $\mathrm{EF}(31.1 \pm 11.9 \%$ and $33.0 \pm 12.9 \%$, respectively), but there was no statistically significant difference between euthyroid, hypothyroid, or hyperthyroid patients in terms of a change in $\mathrm{EF}$ from baseline at either time point $(\mathrm{P}>0.10)$ [2]. It was demonstrated that abnormal thyroid status was nonetheless still associated with increased risk of mortality; hypothyroid and hyperthyroid states were associated with $58 \%$ and $85 \%$ increases in relative risk of death compared to euthyroid state ( $\mathrm{P}<0.0001$ for hypothyroid and $\mathrm{P}=0.0048$ for hyperthyroid vs. euthyroid) [2].

As discussed earlier, excess TH can eventually lead to a decrease in TPR and increase in blood volume, resulting in increased contractility and cardiac output. To compensate for this increased level of stress, it is thought that the heart undergoes a remodeling process. This process is characterized by myocyte enlargement and extracellular matrix deposition [17]. During the initial phase of hyperthyroidism, compensation occurs by proportional growth in both myocyte and myocyte cross sectional area $[17,28]$. This on-going sustained hemodynamic load, however, can overburden the compensatory ability of the heart and result in dilated ventricles with increased myocyte length, without a further increase in relative LVPW thickness [17]. This overall can lead to dilated heart failure. In the acute setting of hyperthyroidism, left ventricle (LV) fibrosis has not been well shown. A study conducted by Weltman and colleagues, using hamster models, used trichrome staining to confirm that prolonged hyperthyroidism, on the other hand, was associated with increased LV fibrosis. They further observed severe relaxation impairment and systolic dysfunction [17].

The Framingham study contradicted these findings, demonstrating that serum TSH concentration was not related to LV end diastolic dimensions, LV wall thickness, LV mass, fractional shortening, or left atrial size [29]. It was shown however, that low serum TSH concentrations $(<0.5 \mathrm{mIU} / \mathrm{L})$ were associated with increased LV contractility at rest in women only, as assessed by echocardiographic LV fractional shortening [29]. Furthermore, in overt hyperthyroidism it has been shown that there was an increased incidence of LV hypertrophy [29, 30], along with increased LVEFs and contractility [29].

Another study by Siu et al demonstrated $6 \%$ of patients 
with hyperthyroidism developed heart failure symptoms; strikingly, half of the patients who presented with heart failure were also found to have a normal EF [27]. It is hypothesized that their symptoms may reflect congestion due to increased sodium and fluid retention, secondary to hyperthyroidism, or may have been caused by tachyarrhythmia-induced diastolic heart failure [27]. These patients responded well to diuretics and rate control with beta-blocker therapy.

As stated earlier, the exact mechanism of how hyperthyroidism can result in left ventricular systolic dysfunction remains unclear. It has been postulated that chronic persistent tachycardia and atrial fibrillation play a role; however, in the study conducted by Siu et al, there was no significant difference seen in the duration of symptoms of hyperthyroidism, incidence of atrial fibrillation, and heart rate between patients with and without systolic dysfunction [27]. In the patients found to have left ventricular dysfunction upon presentation, sustained tachyarrhythmia and atrial fibrillation contributed to the dysfunction, and the heart function recovered following initiation of rate or rhythm control [27]. In the remaining onethird of patients with left ventricular dysfunction at presentation, despite successful rate or rhythm control of tachyarrhythmia, there was only a partial recovery of systolic dysfunction. This suggests additional mechanisms may contribute to the left ventricular dysfunction seen in these patients [27].

Moreover, hyperthyroid patients may present with heart failure symptoms and may be incorrectly labeled as high output heart failure. Unlike true heart failure, which typically has decreased cardiac contractility, these patients typically have increased cardiac output and increased contractility [1]. With thyrotoxic cardiomyopathy, the toxic effects of $\mathrm{TH}$ cause alterations in myocyte energy production, intracellular metabolism, and myofibril contractile function, which results in myocardial damage [1]. The main findings in this condition are left ventricular hypertrophy, arrhythmias, dilated cardiomyopathy, heart failure, $\mathrm{PAH}$, and diastolic dysfunction $[1,24]$. Although symptoms and signs of congestive heart failure are seen in patients with hyperthyroidism, dilated cardiomyopathy and impaired left ventricular systolic function are rarely reported $(<1 \%)$ [27]. While most studies have shown an improvement in left ventricular function following treatment of hyperthyroidism, a proportion of studies have contrarily shown a persistence of dilated cardiomyopathy [27]. One such study by Sui et al was the first systemic study to show persistent dilated cardiomyopathy developed in $1 \%(6 / 519$ patients) of patients with primary hyperthyroidism [27]. Up to one-third of the patients with left ventricular systolic dysfunction at presentation had persistent dilated cardiomyopathy on long-term follow-up [27]. This finding highlights the importance of long-term follow-up in patients with hyperthyroidism and concomitant cardiac dysfunction.

In summary, animal models have clearly demonstrated a correlation between left ventricular dysfunction and fibrosis, and untreated hyperthyroidism. As most human patients do not remain in a prolonged hyperthyroid state, it is difficult to consistently show a correlation between the two entities in human subjects. As mentioned, some studies have demonstrated no change in EF, while other studies have shown acute changes in systolic or diastolic function, with or without an underlying rhythm abnormality. Further research is warranted to clarify the precise mechanism driving cardiac dysfunction in a hyperthyroid state. It is clear, however, that proper management includes conversion to a euthyroid state, heart rate control, and initiation of diuretics. Studies have shown this regimen to lead to improved patient outcomes.

\section{Hyperthyroidism and Brain Natriuretic Peptide (BNP)}

Prior studies have also shown a correlation between BNP levels and thyroid levels in patients without heart failure or cardiac disease $[31,32]$. BNP level was found to be 4-fold higher in patients with hyperthyroidism than in patients in a euthyroid state. This association has not been well studied. It is postulated that THs may stimulate the secretion of BNP, in addition to the main physiologic process: stimulation via stretching of the atrial myocardial tissue. Prior studies have shown that free T3 hormone directly stimulates the secretion of BNP from myocardial cells by increasing gene expression [31, 33, 34].

\section{Hyperthyroidism and the Right Ventricle}

Multiple reports have shown roughly $20 \%$ of patients with pulmonary hypertension have some form of thyroid abnormality $[1,35]$. It is postulated that an increase in pressure in the left atrium leads to an increased pressure in the pulmonary veins, which may stimulate baroreceptors and induce contraction of the arterioles. This results in an increase in pulmonary artery pressure with increased right ventricular load. The increase in right ventricular load increases strain, which further elevates the PAH. The mechanism of PAH and hyperthyroidism is still not well studied, but with proper treatment and a reversal to a euthyroid state, there is typically a significant improvement in right ventricular overload and pulmonary arterial pressure [1, 36]. Therefore, it is beneficial to assess for hyperthyroidism in all patients with $\mathrm{PAH}$.

\section{Subclinical Thyroid Dysfunction and Cardio- vascular System}

Overt hyperthyroidism has been linked to cardiac conditions, and similarly, subclinical thyroid states have shown their own association. Subclinical thyroid abnormalities are characterized as having an abnormal TSH level and normal FT4 level. This is more common in older patients, with an overall prevalence of $10 \%$ for subclinical hypothyroidism and $0.7-3.2 \%$ for subclinical hyperthyroidism $[37,38]$. It has been shown that even subclinical thyroid conditions can be linked to cardiac dysfunction, and some studies have demonstrated that T4 replacement can improve cardiac function, even in patients with subclinical hypothyroidism $[39,40]$. There are a limited number of known studies, however, discussing the association between subclinical thyroid dysfunction and heart failure in particular.

One population-based analysis of adults (ages 70 - 79) illustrated an association between subclinical thyroid dysfunc- 
tion and heart failure episodes. In this study, patients with TSH levels $>7 \mathrm{mU} / \mathrm{L}$ had greater than a 2-fold risk of heart failure episodes, compared to that of euthyroid subjects [39]. A second study supported these findings, with the addition of echocardiographic findings. This trial consisted of 3,044 adults, age greater than 65 years, initially free of heart failure, who were followed over a mean of 12 years. Changes in cardiac function were analyzed both in the patients with subclinical hypothyroidism and subclinical hyperthyroidism [39]. Over the 12 years, 736 patients out of 3,044 were found to develop heart failure symptoms. Patients with TSH levels greater than 10 $\mathrm{mU} / \mathrm{L}$ were found to have a greater incidence of heart failure versus euthyroid participants ( $\mathrm{P}$ value $=0.01$, and $95 \%$ confidence interval of 1.05 - 3.34) [39]. These findings were more prominent and statistically significant in patients with subclinical hypothyroidism rather than subclinical hyperthyroidism. The baseline peak E velocity to measure diastolic dysfunction was higher in those with TSH $>10$ compared to euthyroid participants $(\mathrm{P}$ value $=0.002)$ [39]. These patients were also found to have an increase in left ventricular mass, while other echocardiographic measurements remained unchanged. The patients with subclinical hyperthyroidism and thus TSH levels of 4.5 - 9.9 did not demonstrate a statistically significant increase in heart failure [39].

Most recently, a pooled analysis of six prospective studies examining data from 25,390 participants was conducted to determine the effects of subclinical thyroid dysfunction (with euthyroid defined as TSH 0.45 - $4.49 \mathrm{mIU} / \mathrm{L}$, subclinical hypothyroid as TSH 4.5 - $19.9 \mathrm{mIU} / \mathrm{L}$ and subclinical hyperthyroidism as TSH $<0.45 \mathrm{mIU} / \mathrm{L}$; both with normal FT4 levels) [37]. In this study, the risk of heart failure events was found to be increased with both higher and lower TSH levels, particularly when TSH $>10 \mathrm{mIU} / \mathrm{L}$ and for $\mathrm{TSH}<0.10 \mathrm{mIU} / \mathrm{L}$ (P value for quadratic pattern $<0.01$ ) [37]. The results of this study are notably in contrast to the results of the aforementioned study.

The Famingham Heart study, which included 4,331 patients, did not show a link between subclinical hypothyroidism and increased risk for cardiovascular disease or mortality [2]. In contrast, a study by Tseng et al [41] found that subclinical hypothyroid was associated with increased risk for all-cause and cardiovascular mortality. This study included 115,746 adult patients in Taiwan from 1998 to 1999.

Physicians should be aware of the potential cardiac complications associated with subclinical thyroid dysfunction, mainly hypothyroidism. Subclinical thyroid dysfunction has been well associated in several studies with systolic and diastolic cardiac dysfunction. Importantly, T4 replacement has shown improved measurements of cardiac function in subjects with subclinical hypothyroidism [37, 40]. Elevated TSH levels, even in patients with subclinical hypothyroidism, has been correlated with a decrease in stroke volume, a decrease in cardiac index, and an increase in systemic vascular resistance [37, 42]. Nevertheless, no large-scale studies have been conducted to further support these findings. In terms of subclinical hyperthyroidism, several studies have demonstrated an increased average heart rate, left ventricular mass, and impaired diastolic function compared to overt hyperthyroidism [37, 40]. Subclinical hyperthyroidism has also been correlated with higher rates of atrial fibrillation $[37,43,44]$. This correlation may explain why cardiac dysfunction has been found in patients with subclinical rather than overt hyperthyroidism.

\section{Conclusions}

Guidelines for heart failure produced by the American College of Cardiology and the American Heart Association [2, 45] support conducting thyroid function tests in patients with heart failure to determine if thyroid dysfunction may be a primary contributor to heart failure. In patients with a hyperthyroid state, the importance of prompt diagnosis and treatment of cardiac dysfunction is vital. Anti-thyroid medications are often indicated for the management of thyroid function; however, it may require several weeks to induce a euthyroid state. Definitive treatment with radioactive iodine ablation or thyroidectomy may also be considered to rapidly recover cardiac function [1]. Several studies have validated a reversal of echocardiographic abnormalities in these patients, once a euthyroid state has been established [1, 46, 47]. Additional treatment may include beta-adrenergic blockade to reduce heart rate, and diuretics to improve congestive symptoms [1]. Overall, there has been mixed findings when studying patients with hyperthyroidism and heart failure. A search of the literature demonstrated a clear correlation between hypothyroidism, and even subclinical hypothyroidism, and cardiac dysfunction. Further research is warranted to elucidate the long-term implications of a hyperthyroid state on the cardiovascular system, as well as to clarify the mechanism for the demonstrated effects, whether indirectly via the vascular system, directly by atrial fibrillation or tachyarrhythmia, or via myocyte remodeling and dysfunction.

\section{Acknowledgments}

The authors of this report would like to acknowledge the assistance of the Department of Cardiology at University of Tennessee-Chattanooga.

\section{Financial Disclosure}

The authors have no financial or proprietary interest in the subject matter of this article.

\section{Conflict of Interest}

There are no competing or conflicting interests associated with this article.

\section{Author Contributions}

Each author has individually been involved in and has made substantial contributions to conception and design, acquisition of data, or analysis and interpretation of data. Participants have helped draft the manuscript and revise it critically 
for important intellectual content and have given approval of version to be published. Rafay Khan initiated the project and obtained resources and citations with distribution of the assignments, completed abstract, participated with review and data collection of each topic and section of the review article; was in charge of majority of the work including proofreading and submission; added in also section on Hyperthyroidism and the Right Ventricle with final edit; was in charge of presenting review article thought process and idea to the Department of Cardiology at University of Tennessee, was in charge of discussing project with mentor Dr. Patel, as well as organizing the author sections and contributions; and made breakdown of section categories and specified to each author requirements of each sub-heading. Saad Sikanderkhel contributed to introduction and conclusion, as well as proofreading entire review article. Junhong Guicontributed to the section of TH Physiological Effects on Vasculature and the following section TH Effects on Cardiovascular Physiology, as well as final proofread and data collection. Abdul-Razaq Adeniyi contributed to the sections of TH Physiological Effects on Vasculature and TH Effects on Cardiovascular Physiology with Dr. Gui, as well as final proofread and citation. Kimberly O'Dell, as main review person for correction of grammar and organizing citations, also helped assist write and complete abstract and conclusion segments with Dr. Khan. Marc Ericson and Juan Malpartida were in charge of resource and citation collection from PubMed, along with completion of the subsection of A Brief Look at Hypothyroidism and Cardiology. Zarmina Mufti completed with oversight from Dr. Khan and assistance with colleague and intern Taiba Khan for section titled: An Overview of Hyperthyroidism and Cardiovascular Entities; and also played role in final proofread. Taiba Khan completed with oversight from Dr. Khan and assistance with Dr. Zarmina Mufti for section titled: An Overview of Hyperthyroidism and Cardiovascular Entities. Harris Mufti was supervised by Dr. Al-Adwan to help complete extensive section titled: Hyperthyroidism and Heart Failure, which laid the foundation of the hyperthyroidism paper; and was in charge of this section's final edit and citations as well. Saif Aldeen Al-Adwan contributed majority of section titled Hyperthyroidism and Heart Failure and with final citation. Diana Alvarez was given role to proofread and edit final submission of section Hyperthyroidism and Heart Failure, which was completed by Dr. Al-Adwan and Dr. Harris Mufti, and was also the author of edited section and contribution of section titled: Hyperthyroidism and BNP. Joshua Davis along with Joseph Pendley completed section titled: Subclinical Thyroid Dysfunction and Cardiovascular System. Dharmendra Patel was lead mentor and attending physician/ program director in charge of oversight of the project along with Dr. Khan; reviewed PubMed resources with Dr. Khan and helped formulate thought process with each section; and also was involved in presenting the concept to University of Tennessee Cardiology Department along with the final review.

\section{Data Availability}

The authors declare that data supporting the findings of this study are available within the article.

\section{References}

1. Osuna PM, Udovcic M, Sharma MD. Hyperthyroidism and the Heart. Methodist Debakey Cardiovasc J. 2017;13(2):60-63.

2. Mitchell JE, Hellkamp AS, Mark DB, Anderson J, Johnson GW, Poole JE, Lee KL, et al. Thyroid function in heart failure and impact on mortality. JACC Heart Fail. 2013;1(1):48-55.

3. Helfand M, Force USPST. Screening for subclinical thyroid dysfunction in nonpregnant adults: a summary of the evidence for the U.S. Preventive Services Task Force. Ann Intern Med. 2004;140(2):128-141.

4. Canaris GJ, Manowitz NR, Mayor G, Ridgway EC. The Colorado thyroid disease prevalence study. Arch Intern Med. 2000;160(4):526-534.

5. Jessup M, Abraham WT, Casey DE, Feldman AM, Francis GS, Ganiats TG, Konstam MA, et al. 2009 focused update: ACCF/AHA Guidelines for the Diagnosis and Management of Heart Failure in Adults: a report of the American College of Cardiology Foundation/American Heart Association Task Force on Practice Guidelines: developed in collaboration with the International Society for Heart and Lung Transplantation. Circulation. 2009;119(14):19772016.

6. Grais IM, Sowers JR. Thyroid and the heart. Am J Med. 2014;127(8):691-698.

7. Klein I, Ojamaa K. Thyroid hormone and the cardiovascular system. N Engl J Med. 2001;344(7):501-509.

8. Fukuyama K, Ichiki T, Takeda K, Tokunou T, Iino N, Masuda S, Ishibashi M, et al. Downregulation of vascular angiotensin II type 1 receptor by thyroid hormone. Hypertension. 2003;41(3):598-603.

9. Bussemaker E, Popp R, Fisslthaler B, Larson CM, Fleming I, Busse R, Brandes RP. Hyperthyroidism enhances endothelium-dependent relaxation in the rat renal artery. Cardiovasc Res. 2003;59(1):181-188.

10. Carrillo-Sepulveda MA, Ceravolo GS, Fortes ZB, Carvalho MH, Tostes RC, Laurindo FR, Webb RC, et al. Thyroid hormone stimulates NO production via activation of the PI3K/Akt pathway in vascular myocytes. Cardiovasc Res. 2010;85(3):560-570.

11. Tang YD, Kuzman JA, Said S, Anderson BE, Wang X, Gerdes AM. Low thyroid function leads to cardiac atrophy with chamber dilatation, impaired myocardial blood flow, loss of arterioles, and severe systolic dysfunction. Circulation. 2005;112(20):3122-3130.

12. Savinova OV, Liu Y, Aasen GA, Mao K, Weltman NY, Nedich BL, Liang Q, et al. Thyroid hormone promotes remodeling of coronary resistance vessels. PLoS One. 2011;6(9):e25054.

13. Prisant LM, Gujral JS, Mulloy AL. Hyperthyroidism: a secondary cause of isolated systolic hypertension. J Clin Hypertens (Greenwich). 2006;8(8):596-599.

14. Chang HC. The Blood Volume in Hyperthyroidism. J Clin Invest. 1931;10(3):475-487.

15. Ojamaa K, Kenessey A, Klein I. Thyroid hormone regulation of phospholamban phosphorylation in the rat heart. 
Endocrinology. 2000;141(6):2139-2144.

16. Shenoy R, Klein I, Ojamaa K. Differential regulation of SR calcium transporters by thyroid hormone in rat atria and ventricles. Am J Physiol Heart Circ Physiol. 2001;281(4):H1690-1696.

17. Weltman NY, Wang D, Redetzke RA, Gerdes AM. Longstanding hyperthyroidism is associated with normal or enhanced intrinsic cardiomyocyte function despite decline in global cardiac function. PLoS One. 2012;7(10):e46655.

18. Kahaly GJ, Dillmann WH. Thyroid hormone action in the heart. Endocr Rev. 2005;26(5):704-728.

19. Yen PM, Ando S, Feng X, Liu Y, Maruvada P, Xia X. Thyroid hormone action at the cellular, genomic and target gene levels. Mol Cell Endocrinol. 2006;246(1-2):121-127.

20. Bassett JH, Harvey CB, Williams GR. Mechanisms of thyroid hormone receptor-specific nuclear and extra nuclear actions. Mol Cell Endocrinol. 2003;213(1):1-11.

21. Kuzman JA, Gerdes AM, Kobayashi S, Liang Q. Thyroid hormone activates Akt and prevents serum starvationinduced cell death in neonatal rat cardiomyocytes. J Mol Cell Cardiol. 2005;39(5):841-844.

22. Kenessey A, Ojamaa K. Thyroid hormone stimulates protein synthesis in the cardiomyocyte by activating the Akt-mTOR and p70S6K pathways. J Biol Chem. 2006;281(30):20666-20672.

23. Kruger M, Sachse C, Zimmermann WH, Eschenhagen T, Klede S, Linke WA. Thyroid hormone regulates developmental titin isoform transitions via the phosphatidylinositol-3-kinase/ AKT pathway. Circ Res. 2008;102(4):439447.

24. Vargas-Uricoechea H, Bonelo-Perdomo A, Sierra-Torres $\mathrm{CH}$. Effects of thyroid hormones on the heart. Clin Investig Arterioscler. 2014;26(6):296-309.

25. Nabbout LA, Robbins RJ. The cardiovascular effects of hyperthyroidism. Methodist Debakey Cardiovasc J. 2010;6(2):3-8.

26. Heeringa J, Hoogendoorn EH, van der Deure WM, Hofman A, Peeters RP, Hop WC, den Heijer M, et al. Highnormal thyroid function and risk of atrial fibrillation: the Rotterdam study. Arch Intern Med. 2008;168(20):22192224.

27. Siu CW, Yeung CY, Lau CP, Kung AW, Tse HF. Incidence, clinical characteristics and outcome of congestive heart failure as the initial presentation in patients with primary hyperthyroidism. Heart. 2007;93(4):483-487.

28. Campbell SE, Gerdes AM. Regional changes in myocyte size during the reversal of thyroid-induced cardiac hypertrophy. J Mol Cell Cardiol. 1988;20(5):379-387.

29. Pearce EN, Yang Q, Benjamin EJ, Aragam J, Vasan RS. Thyroid function and left ventricular structure and function in the Framingham Heart Study. Thyroid. 2010;20(4):369-373.

30. Dorr M, Wolff B, Robinson DM, John U, Ludemann J, Meng W, Felix SB, et al. The association of thyroid function with cardiac mass and left ventricular hypertrophy. J Clin Endocrinol Metab. 2005;90(2):673-677.

31. Kishida C, Naito R, Kasuya H, Kaneko T, Yabe K, Kakihara M, Shimai R, et al. Heart Failure with Hyperthyroidism Demonstrating Discrepancy between the Clini- cal Course and B-type Natriuretic Peptide Levels. Intern Med. 2018;57(12):1747-1749.

32. Ertugrul DT, Gursoy A, Sahin M, Unal AD, Pamuk B, Berberoglu Z, Ayturk S, et al. Evaluation of brain natriuretic peptide levels in hyperthyroidism and hypothyroidism. J Natl Med Assoc. 2008;100(4):401-405.

33. Kohno M, Horio T, Yasunari K, Yokokawa K, Ikeda M, Kurihara N, Nishizawa Y, et al. Stimulation of brain natriuretic peptide release from the heart by thyroid hormone. Metabolism. 1993;42(8):1059-1064.

34. Liang F, Webb P, Marimuthu A, Zhang S, Gardner DG. Triiodothyronine increases brain natriuretic peptide (BNP) gene transcription and amplifies endothelin-dependent BNP gene transcription and hypertrophy in neonatal rat ventricular myocytes. J Biol Chem. 2003;278(17):1507315083.

35. Vallabhajosula S, Radhi S, Cevik C, Alalawi R, Raj R, Nugent $\mathrm{K}$. Hyperthyroidism and pulmonary hypertension: an important association. Am J Med Sci. 2011;342(6):507512.

36. Sugiura T, Yamanaka S, Takeuchi H, Morimoto N, Kamioka M, Matsumura Y. Autoimmunity and pulmonary hypertension in patients with Graves' disease. Heart Vessels. 2015;30(5):642-646.

37. Gencer B, Collet TH, Virgini V, Bauer DC, Gussekloo J, Cappola AR, Nanchen D, et al. Subclinical thyroid dysfunction and the risk of heart failure events: an individual participant data analysis from 6 prospective cohorts. Circulation. 2012;126(9):1040-1049.

38. Biondi B, Cooper DS. The clinical significance of subclinical thyroid dysfunction. Endocr Rev. 2008;29(1):76131.

39. Rodondi N, Bauer DC, Cappola AR, Cornuz J, Robbins J, Fried LP, Ladenson PW, et al. Subclinical thyroid dysfunction, cardiac function, and the risk of heart failure. The Cardiovascular Health study. J Am Coll Cardiol. 2008;52(14):1152-1159.

40. Biondi B, Palmieri EA, Lombardi G, Fazio S. Effects of subclinical thyroid dysfunction on the heart. Ann Intern Med. 2002;137(11):904-914.

41. Tseng FY, Lin WY, Lin CC, Lee LT, Li TC, Sung PK, Huang KC. Subclinical hypothyroidism is associated with increased risk for all-cause and cardiovascular mortality in adults. J Am Coll Cardiol. 2012;60(8):730-737.

42. Galli E, Pingitore A, Iervasi G. The role of thyroid hormone in the pathophysiology of heart failure: clinical evidence. Heart Fail Rev. 2010;15(2):155-169.

43. Cappola AR, Fried LP, Arnold AM, Danese MD, Kuller LH, Burke GL, Tracy RP, et al. Thyroid status, cardiovascular risk, and mortality in older adults. JAMA. 2006;295(9):1033-1041.

44. Sawin CT, Geller A, Wolf PA, Belanger AJ, Baker E, Bacharach P, Wilson PW, et al. Low serum thyrotropin concentrations as a risk factor for atrial fibrillation in older persons. N Engl J Med. 1994;331(19):1249-1252.

45. Hunt SA, Abraham WT, Chin MH, Feldman AM, Francis GS, Ganiats TG, Jessup M, et al. ACC/AHA 2005 Guideline Update for the Diagnosis and Management of Chronic Heart Failure in the Adult: a report of the Ameri- 
can College of Cardiology/American Heart Association Task Force on Practice Guidelines (Writing Committee to Update the 2001 Guidelines for the Evaluation and Management of Heart Failure): developed in collaboration with the American College of Chest Physicians and the International Society for Heart and Lung Transplantation: endorsed by the Heart Rhythm Society. Circulation. 2005;112(12):e154-235.

46. Al-Ghamdi AS, Aljohani N. Graves' thyrotoxicosisinduced reversible cardiomyopathy: a case report. Clin Med Insights Case Rep. 2013;6:47-50.

47. Choudhury RP, MacDermot J. Heart failure in thyrotoxicosis, an approach to management. Br J Clin Pharmacol. 1998;46(5):421-424. 